\title{
Editorial
}

\section{Directed Surface Plasmon Resonance for Hot Carrier Applications}

\author{
Gregory T. Forcherio ${ }^{1, *(D)}$ and Jonathan Boltersdorf $2, * \mathbb{D}$ \\ 1 Electro-Optic Technology Division, Naval Surface Warfare Center, Crane, IN 47522, USA \\ 2 United States Army Research Laboratory, Sensors and Electron Devices Directorate, Adelphi, MD 20783, USA \\ * Correspondence: gregory.forcherio@navy.mil (G.T.F.); jonathan.a.boltersdorf.civ@army.mil (J.B.)
}

Citation: Forcherio, G.T.; Boltersdorf,

J. Directed Surface Plasmon

Resonance for Hot Carrier

Applications. Crystals 2021, 11, 1497.

https://doi.org/10.3390/cryst11121497

Received: 23 November 2021

Accepted: 30 November 2021

Published: 2 December 2021

Publisher's Note: MDPI stays neutral with regard to jurisdictional claims in published maps and institutional affiliations.

Copyright: (c) 2021 by the authors. Licensee MDPI, Basel, Switzerland. This article is an open access article distributed under the terms and conditions of the Creative Commons Attribution (CC BY) license (https:/ / creativecommons.org/licenses/by/ $4.0 /)$.
The Special Issue, entitled Directed Surface Plasmon Resonance for Hot Carrier Applications, is a collection of four original articles centered around harnessing energetically "hot" carriers in tailored plasmonic materials for emergent applications in energy harvesting and sensing.

Non-radiative damping of plasmonic resonances was long thought to be a parasitic limitation to the realization of many plasmon-based technologies. Advances in our conceptual understanding, synthesis capabilities, and instrumentation over the last decade elicited renewed interest in using electrical or chemical probes to extract plasmonic, energetically "hot" carriers prior to their thermalization as a means to drive new photodetection and photochemistry concepts. The realization of these concepts for new technologies necessitates advances in our theoretical infrastructure for the design and optimization of nanoplasmonic materials/morphologies, their associated synthesis techniques, and novel spectroscopies with enhanced spatiotemporal resolution to better understand the dynamic behavior of plasmonic "hot" carriers. Envisioned integration spaces span optoelectronic telecommunication, high-density data storage, chemical catalysis, novel manufacturing methods, energy scavenging, therapeutic and diagnostic medicine, heat management, and uncooled infrared photodetectors.

The thematic cornerstones of this Special Issue were computational and empirical design optimization of nanomaterials with directed plasmonic activity towards solar photocatalysis and infrared photodetection. Aravind et al. [1] leveraged iterative finite difference time domain (FDTD) simulations and coupled mode theory to optimize the electric field enhancement, and thereby the generation rate of "hot" electrons, at the surface of corrugated metal gratings for visible photochemistry. Sarma et al. [2] used an artificial intelligence (AI) inverse design approach to experimentally realize near-perfect absorption for "hot" carrier generation on a meta-surface at telecommunication wavelength. Experimental photooxidation demonstrations using nanoplasmonic catalysts, along with their respective empirical optimizations complemented by numerical calculations, are reported in [3,4] by Gordon et al. and Boltersdorf et al., respectively.

The authors hope that this collection of papers will provide inspiration towards new experiments, insights, and conceptual applications in the field of "hot" carrier plasmonics.

Funding: This effort was supported by the Office of Naval Research Code 312, the Naval Innovative Science and Engineering (NISE) program, and the DEVCOM-Army Research Laboratory.

Acknowledgments: The authors would like to thank the Crystals Editorial Office for their timely communication, support, and opportunity in developing this Special Issue, as well as each author who contributed.

Conflicts of Interest: The authors declare no conflict of interest. 


\section{References}

1. Aravind, I.; Wang, Y.; Cai, Z.; Shen, L.; Zhao, B.; Yang, S.; Wang, Y.; Dawlaty, J.M.; Gibson, G.N.; Guignon, E.; et al. Hot Electron Plasmon-Resonant Grating Structures for Enhanced Photochemistry: A Theoretical Study. Crystals 2021, 11, 118. [CrossRef]

2. Sarma, R.; Goldflam, M.; Donahue, E.; Pribisova, A.; Gennaro, S.; Wright, J.; Brener, I.; Briscoe, J. Optimization and Predication of Spectral Response of Metasurfaces Using Artificial Intelligence. Crystals 2020, 10, 1114. [CrossRef]

3. Gordon, W.; Balboa, A.; Giles, S.; Epshteyn, A.; Ávalos-Ovando, O.; Govorov, A.; McEntee, M.; Baturina, O. Visible Light-Induced Reactivity of Plasmonic Gold Nanoparticles Incorporated into $\mathrm{TiO}_{2}$ Matrix towards 2-Chloroethyl Ethyl Sulfide. Crystals 2021, 11, 659. [CrossRef]

4. Boltersdorf, J.; Leff, A.C.; Forcherio, G.T.; Baker, D.R. Plasmonic Au-Pd Bimetallic Nanocatalysts for Hot-Carrier-Enhanced Photocatalytic and Electrochemical Ethanol Oxidation. Crystals 2021, 11, 226. [CrossRef] 